\title{
de Sitter minima from M-theory and string theory
}

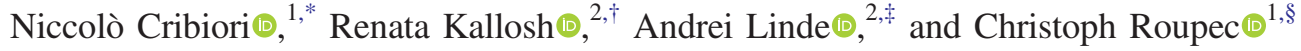 \\ ${ }^{1}$ Institute for Theoretical Physics, TU Wien, Wiedner Hauptstrasse 8-10/136, A-1040 Vienna, Austria \\ ${ }^{2}$ Stanford Institute for Theoretical Physics and Department of Physics, Stanford University, \\ Stanford, California 94305, USA
}

(Received 25 December 2019; accepted 29 January 2020; published 19 February 2020)

\begin{abstract}
We study M-theory compactification on $\mathbb{T}^{7} / \mathbb{Z}_{2}^{3}$ in the presence of a seven-flux, metric fluxes, and KK monopoles. The effective four-dimensional supergravity has seven chiral multiplets whose couplings are specified by the $G_{2}$-structure of the internal manifold. We supplement the corresponding superpotential by a KKLT type nonperturbative exponential contribution for all, or for some of the seven moduli, and find a discrete set of supersymmetric Minkowski minima. We also study type IIA and type IIB string theory compactified on $\mathbb{T}^{6} / \mathbb{Z}_{2}^{2}$. In type IIA, we use a six-flux, geometric fluxes, and nonperturbative exponents. In type IIB theory, we use $\mathrm{F}$ and $\mathrm{H}$ fluxes, and nongeometric $\mathrm{Q}$ and $\mathrm{P}$ fluxes, corresponding to consistently gauged supergravity with certain embedding tensor components, without nonperturbative exponents. Also in these situations, we produce discrete Minkowski minima. Finally, to construct dS vacua starting from these Minkowski progenitors, we follow the procedure of mass production of dS vacua.
\end{abstract}

DOI: 10.1103/PhysRevD.101.046018

\section{INTRODUCTION}

In $[1,2]$, we introduced a method to construct de Sitter minima, starting from Minkowski minima in type IIA and type IIB string theory. Here, we apply this method in the context of M-theory and string theory. All of our models here have seven complex scalars, which are coordinates of the coset space $\left[\frac{S L(2, \mathbb{R})}{S O(2)}\right]^{7}$.

We begin with moduli stabilization in M-theory on a seven-manifold with $G_{2}$-structure, namely, the twisted seven-torus. The starting point is the compact manifold with $\mathbb{Z}_{2} \times \mathbb{Z}_{2} \times \mathbb{Z}_{2} \subset G_{2}$ holonomy that is obtained as the toroidal orbifold of the form $X_{7}=\mathbb{T}^{7} / \mathbb{Z}_{2} \times \mathbb{Z}_{2} \times \mathbb{Z}_{2}$ [3-6]. We make the quotient nonsingular by a choice of a free orbifold action. ${ }^{1}$ The Betti numbers of $X_{7}$ are $\left(b_{0}, b_{1}, b_{2}, b_{3}\right)=(1,0,0,7)$. This theory is identified with the maximal rank reduction on the seven-torus and leads directly to $4 \mathrm{~d} \mathcal{N}=1$ supergravity with seven moduli.

\footnotetext{
*niccolo.cribiori@tuwien.ac.at

†kallosh@stanford.edu

*alinde@stanford.edu

\$christoph.roupec@tuwien.ac.at

${ }^{1}$ We are grateful to A. Braun for explaining this and related issues of $G_{2}$-structures to us.
}

Published by the American Physical Society under the terms of the Creative Commons Attribution 4.0 International license. Further distribution of this work must maintain attribution to the author(s) and the published article's title, journal citation, and DOI. Funded by SCOAP.
Then the twisting is introduced and can be interpreted as a Scherk-Schwarz reduction on the original torus. To derive the twisted seven-torus model from M-theory, it was proposed in [3] how to generalize the action of 11d supergravity to its "democratic form", namely, a pseudoaction where the potentials and the dual curvatures appear at the same time. In 10d, this type of supergravity pseudoaction was proposed in [7]. The pseudoaction allows one to identify the superpotentials in $4 \mathrm{~d}$ supergravity, originating from M-theory on twisted seven-tori. Following [3,5], below we discuss such superpotentials and use them to construct dS minima with all moduli stabilized. Another derivation of an effective $4 \mathrm{~d}$ supergravity theory could also be done using the duality-symmetric $11 \mathrm{~d}$ supergravity action coupled to M-branes [8].

M-theory on a generalized twisted seven-torus was proposed and studied in [5,9], following the corresponding beyond twisted tori constructions in 10d, given in [10]. In particular, the idea in [10] was to introduce Kaluza-Klein monopoles KK5 and KKO5-planes, which allow one to consistently relax some restrictions, known as tadpole conditions. Then, in [5,9], an analogous construction was introduced and studied in M-theory. A "beyond twisted tori" construction was presented by allowing the presence of KK6 monopoles and KKO6-planes.

The purpose of this article is to use M-theory on the generalized twisted seven-torus to identify some relatively simple discrete supersymmetric Minkowski vacua in which all of the 14 real scalars are stabilized. In turn, these vacua can be used to stabilize all of the 14 moduli in dS minima, 
following the mechanism of mass production of dS vacua $[1,2]$. This mechanism is applicable to any M-theory/string theory motivated superpotential satisfying certain conditions. However, all examples given in [1,2] were based on the KL-type racetrack superpotentials containing at least two nonperturbative exponential terms for each of the moduli [11].

In this paper we will show that, by taking into account polynomial flux terms in superpotentials originating from M-theory/string theory, one can achieve dS vacuum stabilization in models with a single exponent for each field. Alternatively, by including additional flux contributions, we can stabilize $\mathrm{dS}$ vacua in models where only some of the moduli have exponential terms in the superpotentials. Some of these M-theory models have also an interpretation as type IIA models compactified on $\mathbb{T}^{6} / \mathbb{Z}_{2}^{2}$ with fluxes.

Finally, we will present a particular class of models in type IIB string theory, describing the seven moduli compactified on $\mathbb{T}^{6} / \mathbb{Z}_{2}^{2}$ with fluxes. The origin of one of the nongeometric fluxes in this model is subtle: it was conjectured in [12] to be present, based on the S-duality of the theory, once the geometric flux is introduced. We show that in this model one can construct stable dS vacua without using any nonperturbative exponential contribution in the superpotential.

\section{GENERALIZED TWISTED SEVEN-TORUS}

Following the discussion in $[3,5]$, where the sevenmoduli model was derived from M-theory, we take the Kähler potential for the seven chiral superfields $\Phi^{i}$ to be ${ }^{2}$

$$
K=-\sum_{i=1}^{7} \log \left(-\mathrm{i}\left(\Phi^{i}-\bar{\Phi}^{i}\right)\right) .
$$

The superpotential derived in [3,5] has the generic form $W_{\text {pert }}=g_{7}+G_{i} \Phi^{i}+\frac{1}{2} M_{i j} \Phi^{i} \Phi^{j}$. In the present work, we will use this superpotential, with two additional modifications. First, we set $G_{i}=0$, in order to have only constant and quadratic terms in the moduli. Second, we add to this superpotential a KKLT-type nonperturbative exponential term. Therefore, the resulting $W$ is

$$
W=g_{7}+\frac{1}{2} M_{i j} \Phi^{i} \Phi^{j}+\sum_{i=1}^{7} A_{i} e^{\mathrm{i} a_{i} \Phi^{i}}
$$

\footnotetext{
${ }^{2}$ In [13], the seven moduli model was derived from $11 \mathrm{~d}$ supergravity compactified on $S^{7}$. It has the same Kähler potential as in (1); however, the superpotential is different, defined by the regular embedding of $[S U(1,1)]^{7}$ into $E_{7(7)}$ and underlying octonian structure. The anti-de Sitter (AdS) critical points in these models were derived using Machine Learning software. We are grateful to N. Bobev, T. Fischbacher, and K. Pilch for attracting our attention to these constructions.
}

Here, $g_{7}$ is a seven-flux contribution, whereas terms quadratic in the moduli originate from geometric fluxes. ${ }^{3}$ Here, all parameters in $W$ are real. The matrix $M_{i j}$ is symmetric and all of its diagonal elements vanish. Therefore, it has 21 parameters. One could generalize this setting and use racetrack superpotentials, following $[1,2]$. In that case, $\mathrm{dS}$ vacuum stabilization is possible even in the absence of the term $g_{7}+\frac{1}{2} M_{i j} \Phi^{i} \Phi^{j}$. The goal of this paper is to explore alternative possibilities, using no more than a single nonperturbative exponential term for each of the moduli.

The nonperturbative exponential terms might arise from wrapped M2-branes. It was shown in [15] that in M-theory compactified on manifolds of $G_{2}$ holonomy, membranes wrapped on 3-cycles induce nonzero corrections to the superpotential. In the $\mathbb{T}^{7} / \mathbb{Z}_{2}^{3}$ model there are seven 3-cycles. Therefore, one expects exponents in $W$ for each of the seven moduli, where $\operatorname{Im} \Phi_{i}$ are the volumes of these seven 3-cycles.

To find supersymmetric Minkowski vacua, one has to solve the equations $\partial_{i} W=0$ and $W=0$. The first of these equations gives

$$
-\mathrm{i} a_{i} A_{i} e^{\mathrm{i} a_{i} \Phi_{i}}=M_{i j} \Phi^{j}
$$

which can be solved for the coefficients $A_{i}$ of the nonperturbative terms, resulting in

$$
A_{i}=\mathrm{i} a_{i}^{-1} e^{-\mathrm{i} a_{i} \Phi_{i}} M_{i j} \Phi^{j} .
$$

We split $\Phi^{i}=\theta^{i}+i \phi^{i}$ and note that the solution is consistent at $\theta^{i}=0$. Then, we substitute the parameters $A_{i}$ evaluated at the extremum, $\phi^{i}=\phi_{0}^{i}, \theta^{i}=\theta_{0}^{i}=0$, back into the superpotential. After that, we subtract from the expression of $W$ the constant term thus obtained. This allows us to fix the parameter $g_{7}$ and to satisfy also the equation $W=0$. This solves the problem of finding a supersymmetric Minkowski vacuum in the seven-moduli $\Phi^{i}$ model.

Therefore, given a free choice of parameters, following this path one can obtain a supersymmetric Minkowski state. We will often find that the number of free parameters is much greater than the number of equations, which may allow us to omit some of the terms in the superpotential and still obtain a supersymmetric Minkowski vacuum. However, if we want to implement the procedure proposed in [1] for producing dS minima, we have to require additionally that the potential does not have flat directions,

\footnotetext{
${ }^{3}$ The seven-moduli case in [2] is now equivalent to M-theory on a seven-torus $\mathbb{T}^{7} / \mathbb{Z}_{2}^{3}$, in the presence of a seven-flux. The terms quadratic in moduli, coming from twisting of the seventorus in M-theory (or, from geometric fluxes in IIA), were not used in [2], but KL-type double exponents were added to a sevenflux instead. D6/O6 and anti-D6 are a reduction from M-theory to string theory of KK6(KKO6). The relation between D6 and KK monopoles in 11d is known as oxidation; see [14].
} 
or, equivalently, that it has a positive definite mass matrix in the vacuum, corresponding to its second derivatives. The mass matrix in a supersymmetric Minkowski vacuum is

$$
V_{i \bar{\jmath}}^{\mathrm{Mink}}=m_{i k} g^{k \bar{k}} m_{\bar{k} \bar{j}}=e^{K} W_{i k} g^{k \bar{k}} \bar{W}_{\bar{k} \bar{j}}
$$

Therefore, in the seven-moduli model we are considering, flat directions are given by the zero modes of

$$
W_{i j}=\partial_{i} \partial_{j} W=M_{i j}-\delta_{i j} A_{i} a_{i}^{2} e^{\mathrm{i} a_{i} \Phi_{i}},
$$

evaluated in the vacuum. Notice that, if we have exponents in all directions, as in (2), the matrix $W_{i j}$ is a generic symmetric matrix, including nonvanishing diagonal terms. Since $g_{i j}$ is positive definite, one or more zero modes are in fact present in the mass matrix when $\operatorname{det} W_{i j}=0$. However, as we will show in several examples, this is actually a quite restrictive condition, which does not hold in generic models, unless peculiar cancellations occur. Therefore, in general one expects that

$$
\operatorname{det} W_{i j} \neq 0 \text {, }
$$

and no flat directions are present in the mass matrix.

In our previous papers [1,2], where only constant terms in $W$ were present, a KL-type double exponent was necessary for each direction in the moduli space in order to obtain stable solutions. All such models do not have flat directions, by construction. Meanwhile in the new set of models discussed in this paper one may encounter flat directions, but one can eliminate them by adding fluxes. In each of the models to be studied in this paper we found that the flat directions are absent in Minkowski vacua for a broad range of parameters, i.e., no fine tuning is necessary.

Furthermore, by adding more flux contributions, one can eliminate some of the single exponents, and by adding extra contributions from S-dual fluxes, as in (15), one can eliminate all of the exponents, still without flat directions. This is one of the central, most unexpected results of this paper.

In the presentation of our examples, we split the sevenmoduli in a type IIA language, as

$$
\Phi^{i}=\left\{S, T_{I}, U_{J}\right\}, \quad I, J=1,2,3 .
$$

For convenience, we keep the same notation also for type IIB examples in Sec. V. Following also [5], the 21 nonvanishing terms contained in $M_{i j}$, in the case of effective supergravities coming from twisted reductions of M-theory on a $X_{7}=\mathbb{T}^{7} / \mathbb{Z}_{2}^{3}$ orbifold with fluxes, can be represented as:

$$
\begin{aligned}
\frac{1}{2} M_{i j} \Phi^{i} \Phi^{j}= & S b^{K} U_{K}+U_{I} C^{I J} T_{J}+a^{I} \frac{U_{1} U_{2} U_{3}}{U_{I}} \\
& +c^{I} \frac{T_{1} T_{2} T_{3}}{T_{I}}+S d^{K} T_{K} .
\end{aligned}
$$

The 21 entries of $M_{i j}$ are now given in terms of the parameters $a^{I}, b^{K}, c^{I}, d^{K}$, and $C^{I J}$. However, for our purpose of finding discrete supersymmetric Minkowski vacua, it is sufficient to use only some of these terms.

\section{A. Model 1, with S, T, and $U$ exponents}

In this first class of models, we engage only 12 terms in $M_{i j}$ and keep one exponent for each of the seven directions. The resulting superpotential is then

$$
\begin{aligned}
W_{1}= & g_{7}+b^{K} S U_{K}+C^{I J} U_{I} T_{J}+A_{S} e^{\mathrm{i} a_{S} S}+\sum_{I} A_{T_{I}} e^{\mathrm{i} a_{T_{I}} T_{I}} \\
& +\sum_{I} A_{U_{I}} e^{\mathrm{i} a_{U_{I}} U_{I}} .
\end{aligned}
$$

In the vacuum, we have a total of 19 free parameters: $7 a_{i}$, $3 b^{I}$ and 9 parameters $C^{I J}$. Instead of fixing all of them and looking for the minimum of the potential, one can use 8 equations $\partial_{i} W=0$ and $W=0$ to find 8 parameters $g_{7}$ and $A_{i}$ such that these equations are satisfied at a chosen point $\Phi^{i}$ in moduli space, which therefore describes a supersymmetric Minkowski vacuum. This still leaves plenty of free parameters to control the values of masses of all moduli in the vacuum and to ensure that there are no flat directions. As we will show in numerical examples, many options are available, even if one does not engage some of the exponents. We show one explicit example with unconstrained parameters, and another one where the tadpole conditions are satisfied without sources.

\section{B. Model 2, without S exponent}

A second class of models we consider is a subclass of the previous one, in which we set $A_{S}=a_{S}=0$ from the very beginning. In other words, we again use the 12 terms from the $M_{i j}$ matrix and add the exponents in all of the directions but $S$. The superpotential in this case takes the form:

$$
\begin{aligned}
W_{2}= & g_{7}+b^{K} S U_{K}+C^{I J} U_{I} T_{J} \\
& +\sum_{I} A_{T_{I}} e^{\mathrm{i} a_{T_{I}} T_{I}}+\sum_{I} A_{U_{I}} e^{\mathrm{i} a_{U_{I}} U_{I}}
\end{aligned}
$$

Solving $W=0$ and $\partial_{i} W=0$, in order to find a supersymmetric Minkowski solution, will now fix the parameters $g_{7}, A_{T_{I}}, A_{U_{I}}$, together with one of the parameters among $b^{K}$ or $C^{I J}$, in (11). As it turns out, this does not prohibit a solution. Indeed, explicit examples of this class of models are possible. We present one such solution in Sec. IV. 


\section{Model 3, without $U$ exponents}

In the third class of models, we consider 15 terms from the matrix $M_{i j}$ and add the exponents only in four directions, namely $S$ and $T_{I}$. In particular, it turns out that we do not need to add exponents in the $U_{K}$ directions. This is interesting, since in $[2,16]$ such terms were employed in order to facilitate stable dS vacua in type IIA supergravity constructions, in which the only perturbative term in $W$ was a constant flux. Here we find that, by including in $W$ geometric fluxes polynomial in the moduli and looking first for a supersymmetric Minkowski minimum, some of the nonperturbative exponential terms are not required. Therefore, we consider the superpotential

$$
\begin{aligned}
W_{3}= & g_{7}+a^{I} \frac{U_{1} U_{2} U_{3}}{U_{I}}+b^{K} S U_{K}+C^{I J} U_{I} T_{J} \\
& +A_{S} e^{\mathrm{i} a_{S} S}+A_{T_{I}} e^{\mathrm{i} a_{T_{I}} T_{I}},
\end{aligned}
$$

which has 24 parameters. Again, we have to solve the equations $\partial_{i} W=0$ and $W=0$, which will fix 8 parameters in $W$. We are then free to choose the remaining parameters in order to obtain appropriate masses in Minkowski. We present an explicit numerical example of this class of models in Sec. IV.

\section{Model 4, without $T$ and $U$ exponents}

In this fourth class of models, we engage 18 terms from the matrix $M_{i j}$ and add the exponential contribution only in one direction, namely $S$. In particular, in this case we find that there is no need to add exponents in the $T_{I}$ and $U_{K}$ directions. Therefore, the superpotential is

$$
\begin{aligned}
W_{4}= & g_{7}+a^{I} \frac{U_{1} U_{2} U_{3}}{U_{I}}+b^{K} S U_{K}+C^{I J} U_{I} T_{J} \\
& +c^{I} \frac{T_{1} T_{2} T_{3}}{T_{I}}+A_{S} e^{\mathrm{i} a_{S} S},
\end{aligned}
$$

which has 21 parameters. We solve the 8 equations $\partial_{i} W=0$ and $W=0$ once more and fix the remaining free parameters to produce Minkowski vacua without flat directions. We show a numerical realisation of this model in Sec. IV.

\section{GENERALIZED TWISTED SIX-TORUS}

Following $[5,9,10]$, in type IIA string theory compactified on $\frac{\mathbb{W}^{6}}{\mathbb{Z}_{2} \times \mathbb{Z}_{2}}$ one finds that only 15 terms are available, out of the total 21 terms present in M-theory and given in (9). In particular, the last two terms in (9), namely $c^{I} \frac{T_{1} T_{2} T_{3}}{T_{I}}+S d^{K} T_{K}$, with 6 parameters, $c^{I}$ and $d^{K}$, are absent in standard type IIA orientifold constructions. Furthermore, the six-flux $f_{6}$ in type IIA replaces the seven-flux $g_{7}$ of the M-theory models. In the notation of [9], with $a=1,2,3$ and $m=4,5,6$, the 3 terms $a^{I} \frac{U_{1} U_{2} U_{3}}{U_{I}}$ correspond to two-fluxes $F_{a m}$. The 3 terms of the form $b^{I} S U_{I}$ correspond to nongeometric fluxes, with $b^{I}$ defined by $\omega_{m n}{ }^{c}$. Finally, the 9 terms of the form $C^{I J} U_{I} T_{J}$ correspond to nongeometric fluxes, where $C^{I J}$ is defined by $\omega_{b p}{ }^{m}, \omega_{b c}{ }^{a}$. Thus, our Models 1, 2, 3 are also models in type IIA. Instead, our M-theory Model 4 is not related to standard type IIA orientifold constructions, due to the presence of the term $c^{I} \frac{T_{1} T_{2} T_{3}}{T_{I}}$.

The tadpole conditions require spacetime filling sources, such as O6 planes, D6 branes, and KK monopoles, as explained in detail in $[9,10]$. In these cases, the combinations of fluxes $\sum_{I} a^{I} b^{I}$ and $\sum_{J} a^{J} C^{J I}$ (see Table 2 in [9]) do not have to vanish, but can be canceled by specific O6/D6 sources. Similarly, the expressions $b^{I} C^{I J}+b^{J} C^{I I}$ and $C^{I J} C^{J K}+C^{I K} C^{J J}$ do not need to be set to zero, but can be canceled by contributions from (KK5/KKO5) and from (KK5/KKO5)', respectively, where these sources are wrapped on specific internal cycles.

In Models 3 and 4, we need to consider all of these conditions, while in Models 1 and 2 the first two are satisfied automatically, since $a^{I}=0$. The fact that the tadpole conditions can be satisfied in the presence of sources means, as it was already suggested in $[9,10]$, that there is no need to enforce the Jacobi constraints on flux parameters, which would be required in the absence of sources. Our examples will include one case where the tadpole identities are satisfied even without sources, as well as more general cases with sources and relaxed Jacobi constraints.

\section{M-THEORY EXAMPLES}

In this section, we investigate the models described above, in the context of an effective $4 \mathrm{~d} \mathcal{N}=1$ supergravity description and present numerical examples. The Kähler potential, in our conventions, takes the form (1) and the complete superpotential is given in Eq. (2) and Eq. (9). After solving for the supersymmetric Minkowski vacuum and choosing the free parameters such that there are no flat directions, we follow the mass production mechanism $[1,2]$ in order to find a dS solution. We refrain from giving the details of this construction here and choose to present only the independent set of parameters and masses in Minkowski as well as in dS.

One important comment concerns the uplifting procedure, which is well understood in both type IIB as well as type IIA string theory. It is based on pseudocalibrated Dp-branes [17] and results in an equivalent procedure of supplementing $4 d, \mathcal{N}=1$ supergravity by a nilpotent multiplet. ${ }^{4}$ In M-theory, the analogous procedure has not

\footnotetext{
${ }^{4}$ Examples of dS vacua without an uplifting anti-Dp-brane (without a nilpotent multiplet) based on perturbative and nonperturbative contributions are given in [18] and based on higher derivative $R^{4}$ correction in M-theory models in [19].
} 
TABLE I. Our set of chosen parameters for Model 1. Note that $S_{0}$ corresponds to the imaginary part of the modulus, similarly for all of the other moduli. The values of the moduli $U_{I}$ are chosen in this way because, in our conventions, $\operatorname{Im}\left(U_{I}\right)$ corresponds to the volume of the internal manifold, which should be large in IIA. Included are the downshift $\Delta g_{7}$ and uplift parameter $\mu^{4}$ for the mass production procedure.

\begin{tabular}{lccccccc}
\hline \hline$S_{0}$ & 1.0 & $a_{S}$ & 1.0 & $C^{11}$ & 0.11 & $C^{32}$ & 0.32 \\
$T_{1,0}$ & 1.1 & $a_{T_{1}}$ & 1.1 & $C^{12}$ & 0.12 & $C^{33}$ & 0.33 \\
$T_{2,0}$ & 1.2 & $a_{T_{2}}$ & 1.1 & $C^{13}$ & 0.13 & $b^{1}$ & 0.55 \\
$T_{3,0}$ & 1.3 & $a_{T_{3}}$ & 1.1 & $C^{21}$ & 0.21 & $b^{2}$ & 0.60 \\
$U_{1,0}$ & 5.1 & $a_{U_{1}}$ & 0.51 & $C^{22}$ & 0.22 & $b^{3}$ & 0.65 \\
$U_{2,0}$ & 5.2 & $a_{U_{2}}$ & 0.52 & $C^{23}$ & 0.23 & $\Delta g_{7}$ & $5 \times 10^{-3}$ \\
$U_{3,0}$ & 5.3 & $a_{U_{3}}$ & 0.53 & $C^{31}$ & 0.31 & $\mu^{4}$ & $9 \times 10^{-9}$ \\
\hline \hline
\end{tabular}

been worked out in detail yet and it will be a matter of future investigations.

\section{A. Model 1, with S, T, and $U$ exponents}

The superpotential of this model is given in (10). For our first example, we choose to solve the Minkowski conditions $W=0$ and $\partial_{i} W=0$ in terms of the parameters $A_{S}$, $A_{T_{I}}, A_{U_{I}}(I=1,2,3)$, and the seven-flux $g_{7}$. All of the other parameters, as well as the position of the minimum in moduli space, remain free. Then, we choose values for these free parameters in a way that avoids flat directions, which might happen in case of accidental cancellations, for very specific values of the parameters. One possible choice for the free parameters is given in Table I. These parameters lead to a stable, supersymmetric Minkowski vacuum with canonical masses given in Table II.

\section{B. Model 1, with the tadpole condition satisfied without sources}

Another interesting variation of the model with $b^{K}$ and $C^{I J}$ terms is connected to the tadpole conditions, as taken from Table 2 of [9]. Usually the tadpole conditions are satisfied by inclusion of sources. However, we find that, if we include exponents in all directions, we are able to satisfy all of the tadpole conditions without sources in this model. The relevant tadpole conditions, without sources, are:

$$
\begin{aligned}
b^{I} C^{I J}+b^{J} C^{I I} & =0, \\
C^{I J} C^{J K}+C^{I K} C^{J J} & =0, \quad \text { (no summation). }
\end{aligned}
$$

TABLE II. The canonical normalized masses for Model 1. We choose to give only the masses of the moduli, omitting the axions. The behavior follows exactly as described in [2].

\begin{tabular}{lccccccc}
\hline \hline & $m_{1}$ & $m_{2}$ & $m_{3}$ & $m_{4}$ & $m_{5}$ & $m_{6}$ & $m_{7}$ \\
\hline Mk & 0.6421 & 0.4700 & 0.3216 & 0.1757 & 0.1406 & 0.1129 & 0.08219 \\
dS & 0.6427 & 0.4705 & 0.3218 & 0.1758 & 0.1407 & 0.1130 & 0.08227 \\
\hline \hline
\end{tabular}

TABLE III. The canonical normalized masses for Model 1 with all tadpole conditions solved.

\begin{tabular}{cccccccc}
\hline \hline & $m_{1}$ & $m_{2}$ & $m_{3}$ & $m_{4}$ & $m_{5}$ & $m_{6}$ & $m_{7}$ \\
\hline Mk & 0.3006 & 0.1641 & 0.1179 & 0.07467 & 0.06229 & 0.03988 & 0.02517 \\
dS & 0.2997 & 0.1637 & 0.1176 & 0.07449 & 0.06227 & 0.03976 & 0.02513 \\
\hline \hline
\end{tabular}

We choose to solve these conditions in terms of the $C^{I J}$ with $I \neq J$, keeping the other parameters as in Table I. This leads to a stable solution with masses given in Table III.

\section{Model 2, without $\mathrm{S}$ exponent}

It is possible to set $A_{S}=0$ from the very beginning, as given in (11), in order to eliminate the nonperturbative contributions for the S-direction. Then, solving the 8 supersymmetric Minkowski equations for such a reduced model gives a restriction on one of the flux parameters, for example $b^{1}$, besides the 7 parameters $g_{7}, A_{T_{I}}$, and $A_{U_{I}}$. Keeping all of the other parameters the same as in Table I leads to a stable solution, with masses given in Table IV.

\section{Model 3, without $U$ exponents}

The superpotential of this model is given in (12). Compared to Model 1 and Model 2, it contains an additional term $a^{I} \frac{U_{1} U_{2} U_{3}}{U_{I}}$, which allows one to build dS vacua without the U-exponent. When evaluating the conditions for supersymmetric Minkowski vacua, we can now solve for the three parameters $a^{I}$ (these $a^{I}$ parameters should not be confused with the parameters in the exponents, $\left.a_{\Phi_{i}}\right)$. We find a stable dS solution with the same parameters as in Table I and give the masses in Table V.

\section{E. Model 4, without $T$ and $U$ exponents}

The superpotential of Model 4 is defined in (13). Including the terms $c^{I} \frac{T_{1} T_{2} T_{3}}{T_{I}}$, from (13), we find that it is in fact possible to find a Minkowski solution without

TABLE IV. The canonical normalized masses of the moduli for Model 2 without nonperturbative contributions for the $S$ direction.

\begin{tabular}{lccccccc}
\hline \hline & $m_{1}$ & $m_{2}$ & $m_{3}$ & $m_{4}$ & $m_{5}$ & $m_{6}$ & $m_{7}$ \\
\hline Mk & 0.6360 & 0.4629 & 0.3295 & 0.1491 & 0.1225 & 0.09989 & 0.03602 \\
dS & 0.6365 & 0.4633 & 0.3297 & 0.1492 & 0.1226 & 0.09993 & 0.03607 \\
\hline \hline
\end{tabular}

TABLE V. The canonical normalized masses for Model 3, without nonperturbative exponential corrections in the $U$-directions.

\begin{tabular}{lccccccc}
\hline \hline & $m_{1}$ & $m_{2}$ & $m_{3}$ & $m_{4}$ & $m_{5}$ & $m_{6}$ & $m_{7}$ \\
\hline Mk & 0.2569 & 0.2342 & 0.1706 & 0.1424 & 0.1260 & 0.1030 & 0.02566 \\
dS & 0.2572 & 0.2344 & 0.1707 & 0.1425 & 0.1261 & 0.1030 & 0.02565 \\
\hline \hline
\end{tabular}


TABLE VI. The canonical normalized masses for the model with only one exponent, in the $S$-direction.

\begin{tabular}{lllllll}
\hline \hline$m_{1}$ & $m_{2}$ & $m_{3}$ & $m_{4}$ & $m_{5}$ & $m_{6}$ & $m_{7}$ \\
\hline
\end{tabular}

Mk $\begin{array}{llllllll}0.2639 & 0.2520 & 0.1469 & 0.06163 & 0.04579 & 0.03365 & 0.02874\end{array}$ $\begin{array}{lllllllll}\text { dS } & 0.2636 & 0.2513 & 0.1467 & 0.06163 & 0.04565 & 0.03363 & 0.02871\end{array}$

any exponents other than $A_{S} e^{\mathrm{i} a_{S} S}$; i.e., we set $A_{T_{I}}=A_{U_{I}}=0$ for all $I$. Instead of solving for the prefactors of the exponents in the $T$ and $U$ directions, we now obtain the solutions in terms of the parameters $a^{I}$ and $c^{I}$ of the terms quadratic in $U$ and $T$-moduli. Once again, we use the parameters of Table I and obtain the Minkowski and dS masses for the moduli given in Table VI. Once more, we found a stable dS solution after the mass production procedure.

To summarize the results obtained so far, in Model 2, Model 3, and Model 4 we find that quadratic tree-level contributions to the superpotential can take the place of some of the nonperturbative exponential terms that are usually required.

\section{IIB THEORY, GAUGED SUPERGRAVITY AND DS VACUA}

In this section, we continue the investigation of the seven-moduli model with the Kähler potential given in (1). The superpotential $W$ of the type IIB theory $[12,20,21]$ has the following 3 structures: contributions coming from the $F$-flux, from the $H$-flux, and from the $Q$-flux, which are all known fluxes in type IIB string theory. In addition, it was conjectured in [12] that certain $P$-fluxes should be present due to S-duality of string theory. In [20], it was recognized that terms in $W$ of the form coming from the conjectured $P$-fluxes appear naturally as components of gauged supergravity in $4 \mathrm{~d}$, when the embedding tensor procedure is performed consistently.

For our purpose we will keep only terms even in the moduli in the superpotential; namely, we will use

$$
\begin{aligned}
W_{5}= & a_{0}+a^{I} \frac{U_{1} U_{2} U_{3}}{U_{I}}+S\left(b^{I} U_{I}+b_{3} U_{1} U_{2} U_{3}\right) \\
& +T_{K}\left(C^{I K} U_{I}-c^{K} U_{1} U_{2} U_{3}\right) \\
& -S T_{K}\left(d^{K}-D^{I K} \frac{U_{1} U_{2} U_{3}}{U_{I}}\right) .
\end{aligned}
$$

The first, second, third, and fourth line represent the even parts of $F-, H-, Q-$, and $P$ - flux, respectively. We find that the terms with coefficients $b^{I}$ and $D^{I K}$ are not necessary for full stabilization of moduli, in this model. One can use (15), with or without terms proportional to $b^{I}$ and $D^{I K}$, as a new model which does not have non-perturbative exponents in $W$. Thus, we use (15) with $D^{I K}=0$ as a new model which does not have nonperturbative exponents in $W$. As a numerical example, we have found that there is a Minkowski minimum
TABLE VII. The independent parameters for our Model 5. These produce the values for the masses in Table VIII. No particular fine-tuning is necessary.

\begin{tabular}{rrrrrrrrrr}
\hline \hline$b^{1}$ & 0.55 & $C^{11}$ & -0.11 & $C^{21}$ & 0.21 & $C^{31}$ & 0.31 & $d^{1}$ & 5.1 \\
$b^{2}$ & 0.60 & $C^{12}$ & 0.12 & $C^{22}$ & -0.22 & $C^{32}$ & 0.32 & $d^{2}$ & -5.2 \\
$b^{3}$ & 0.65 & $C^{13}$ & 0.13 & $C^{23}$ & 0.23 & $C^{33}$ & -0.33 & $d^{3}$ & 5.3 \\
\hline \hline
\end{tabular}

TABLE VIII. For the IIB model without exponents, where all contributions come from tree-level fluxes, we find these canonical masses for the moduli.

\begin{tabular}{lccccccc}
\hline \hline & $m_{1}$ & $m_{2}$ & $m_{3}$ & $m_{4}$ & $m_{5}$ & $m_{6}$ & $m_{7}$ \\
\hline Mk & 0.5392 & 0.4551 & 0.1037 & 0.06185 & 0.05355 & 0.02389 & 0.01263 \\
dS & 0.5391 & 0.4552 & 0.1036 & 0.06183 & 0.05357 & 0.02381 & 0.01260 \\
\hline \hline
\end{tabular}

without flat directions. This means that we were able to employ this model in order to get a dS minimum, using the technology developed in $[1,2]$.

\section{A. Model 5, without any exponents}

In order to find an explicit example of a dS vacuum from the above model, we again have to solve the Minkowski conditions, $W=0$ and $\partial_{i} W=0$, where $i=S, T_{I}, U_{I}$ with $I=1,2,3$. This will fix 8 of the parameters in (15). We choose, in this case, to solve for the following set: $a_{0}, a^{I}, b_{3}$, and $c^{K}$. For the position in moduli space, the downshift to AdS, $\Delta a_{0}=\Delta g_{7}$, and uplift to dS, we choose the same values as in Table I. These values are supplemented by the ones in Table VII.

We found a stable Minkowski solution and then were able to follow the mass production procedure to obtain a dS vacuum with masses given in Table VIII. We also found that it is easy to change the parameters and still have dS minima, without particular fine-tuning. This model is very interesting since it has only polynomial terms in the superpotential.

\section{DISCUSSION}

M-theory is supposed to unify all of the consistent versions of superstring theory. At low energies it should be approximated by $11 \mathrm{~d}$ supergravity. Furthermore, it should also describe various extended objects, like M2 and M5 branes, KK6 monopoles, and KKO6-planes, such that extended objects of string theory, like Dp-branes and Op-planes, are included. The existence of such a theory was first conjectured by Witten in 1995. Some early papers on M-theory include [22-24] and more information can be found in the books $[14,25]$. A particularly relevant description of M-theory and $4 \mathrm{~d}$ gauged supergravity is given in $[3,5,10,20]$. We are using these models in our construction of $4 \mathrm{~d} \mathrm{dS}$ vacua. The main issue in studies of specific models of dS minima in $4 \mathrm{~d}$ gauged supergravity is their motivation from string theory or M-theory. 
Here we focused on a model where seven complex scalars are coordinates of the coset space $[S L(2$, $\mathbb{R} / S O(2)]^{7}$. This model is available in M-theory and in type IIA and type IIB string theory. As a technical tool for constructing dS minima, we use the method of mass production of $\mathrm{dS}$ vacua proposed in [1,2], based on the possibility to make parametrically small deformations (downshift and uplift) of a supersymmetric Minkowski vacuum state, without flat directions. In all of the cases, the uplift is due to the existence of the pseudocalibrated antiDp-branes in string theory, which in $4 \mathrm{~d}$ supergravity is equivalent to the presence of a nilpotent chiral multiplet [17]. In M-theory, the details of the uplifting procedure need to be investigated. We presented several classes of models with stable $\mathrm{dS}$ vacua, with numerical examples in Models 1-5. In these models a better understanding of the role of the geometric fluxes and tadpole conditions will be required, based on earlier studies of these issues in [26-28].

In all of the models which we studied in M-theory, namely Models 1, 2, 3, 4, we used a superpotential $W$ with polynomial terms in the moduli, of degrees 0 and 2, and a single nonperturbative KKLT-type exponent for some of the moduli, as shown in (9). This is different from the case without terms quadratic in the moduli, where supersymmetric Minkowski vacua without flat directions are possible with KL-type double set of exponents in every moduli direction $[1,2]$. After adding quadratic terms, we found supersymmetric Minkowski vacua without flat directions by engaging a single nonperturbative exponent for each of the 7 moduli, or only for 4 of them, or only for the $S$ field. In all of the models of this kind, namely Models 1, 2, 3, 4, we found locally stable dS minima.
Perhaps the most surprising result is the model in Sec. V, in type IIB string theory, which we call Model 5. Only terms which are even polynomials in moduli, of degree 0,2 , 4 , are present in (15), and no nonperturbative exponents are required. In a model of $4 \mathrm{~d}$ supergravity associated with IIB string theory presented in Sec. V, all of the terms in the Kähler and superpotential are identified with type IIB string theory. The only somewhat unusual term in (15) is $S T_{K} d_{0}^{(K)}$. It was conjectured to be present in type IIB theory in [12], to support $S$-duality. It is interesting that this same term is also present in M-theory in (9), as well as in a consistent gauged supergravity in [20]. We have constructed supersymmetric Minkowski minima without flat directions, and the corresponding $\mathrm{dS}$ minima in this sevenmoduli model.

\section{ACKNOWLEDGMENTS}

We are grateful to I. Bena, J. Blåbäck, A. Braun, G. Dibitetto, M. Grana, L. Martucci, E. Plauschinn, T. Van Riet, N. Warner, T. Wrase, I. Zavala, and other participants of the workshop "de Sitter constructions in String theory" at Saclay, for valuable discussions. R. K. and A. L. are supported by Stanford Institute for Theoretical Physics (SITP) and by the US National Science Foundation Grant No. PHY-1720397, and by the Simons Foundation Origins of the Universe program (Modern Inflationary Cosmology collaboration), and by the Simons Fellowship in Theoretical Physics. N. C. and C. R. are supported by an FWF Grant No. P 302.65. C. R. is grateful to SITP for the hospitality while this work was performed and to the Austrian Marshall Plan Foundation for making his stay at SITP possible.
[1] R. Kallosh and A. Linde, Mass production of type IIA dS Vacua, arXiv:1910.08217.

[2] N. Cribiori, R. Kallosh, A. Linde, and C. Roupec, Mass production of IIA and IIB dS Vacua, arXiv:1912.00027.

[3] G. Dall'Agata and N. Prezas, Scherk-Schwarz reduction of M-theory on G2-manifolds with fluxes, J. High Energy Phys. 10 (2005) 103.

[4] M. J. Duff and S. Ferrara, Four curious supergravities, Phys. Rev. D 83, 046007 (2011).

[5] J.-P. Derendinger and A. Guarino, A second look at gauged supergravities from fluxes in M-theory, J. High Energy Phys. 09 (2014) 162.

[6] S. Ferrara and R. Kallosh, Seven-disk manifold, $\alpha$-attractors, and $B$ modes, Phys. Rev. D 94, 126015 (2016).

[7] E. Bergshoeff, R. Kallosh, T. Ortin, D. Roest, and A. Van Proeyen, New formulations of $D=10$ supersymmetry and D8-O8 domain walls, Classical Quantum Gravity 18, 3359 (2001).
[8] I. A. Bandos, N. Berkovits, and D. P. Sorokin, Duality symmetric eleven-dimensional supergravity and its coupling to $M$-branes, Nucl. Phys. B522, 214 (1998).

[9] J. Blaback, U. Danielsson, and G. Dibitetto, A new light on the darkest corner of the landscape, arXiv:1810.11365.

[10] G. Villadoro and F. Zwirner, Beyond twisted tori, Phys. Lett. B 652, 118 (2007).

[11] R. Kallosh and A. D. Linde, Landscape, the scale of SUSY breaking, and inflation, J. High Energy Phys. 12 (2004) 004.

[12] G. Aldazabal, P. G. Camara, A. Font, and L. E. Ibanez, More dual fluxes and moduli fixing, J. High Energy Phys. 05 (2006) 070.

[13] N. Bobev, T. Fischbacher, and K. Pilch, A new $N=1$ AdS4 Vacuum of Maximal Supergravity, J. High Energy Phys. 01 (2020) 099.

[14] T. Ortin, Gravity and Strings, Cambridge Monographs on Mathematical Physics (Cambridge University Press, Cambridge, England, 2015), http://www.cambridge.org/ 
mw/academic/subjects/physics/theoretical-physics-andmathematical-physics/gravity-and-strings-2nd-edition.

[15] J. A. Harvey and G. W. Moore, Superpotentials and membrane instantons, arXiv:hep-th/9907026.

[16] N. Cribiori, R. Kallosh, C. Roupec, and T. Wrase, Uplifting Anti-D6-brane, J. High Energy Phys. 12 (2019) 171.

[17] R. Kallosh and T. Wrase, dS Supergravity from 10d, Fortsch. Phys. 2018, 1800071 (2018).

[18] J. Blaback, D. Roest, and I. Zavala, De Sitter Vacua from nonperturbative flux compactifications, Phys. Rev. D 90, 024065 (2014).

[19] J. Blaback, U. Danielsson, G. Dibitetto, and S. Giri, Constructing stable de Sitter in M-theory from higher curvature corrections, J. High Energy Phys. 09 (2019) 042.

[20] G. Dibitetto, A. Guarino, and D. Roest, Charting the landscape of $N=4$ flux compactifications, J. High Energy Phys. 03 (2011) 137.

[21] J. Blaback, U. Danielsson, and G. Dibitetto, Fully stable dS vacua from generalized fluxes, J. High Energy Phys. 08 (2013) 054.
[22] E. Witten, Five-branes and M theory on an orbifold, Nucl. Phys. B463, 383 (1996).

[23] M. J. Duff, M theory (The Theory formerly known as strings), Int. J. Mod. Phys. A 11, 5623 (1996).

[24] A. Sen, An Introduction to nonperturbative string theory, in Duality and supersymmetric theories. Proceedings, Easter School, Newton Institute, Euroconference, Cambridge, UK, 1997 (Cambridge University Press, Cambridge, 1999), p. 473, arXiv:hep-th/9802051.

[25] C. V. Johnson, D-branes, Cambridge Monographs on Mathematical Physics (Cambridge University Press, Cambridge, England, 2005), http://books.cambridge.org/ 0521809126.htm.

[26] M. Grana, R. Minasian, M. Petrini, and A. Tomasiello, A Scan for new $N=1$ vacua on twisted tori, J. High Energy Phys. 05 (2007) 031.

[27] M. Grana, R. Minasian, H. Triendl, and T. Van Riet, Quantization problem in Scherk-Schwarz compactifications, Phys. Rev. D 88, 085018 (2013).

[28] E. Plauschinn, Non-geometric backgrounds in string theory, Phys. Rep. 798, 1 (2019). 